\title{
Editorial
}

\section{Actualidad y reto de los sistemas de estimación pronóstica en pacientes críticos}

\author{
S. MAS FONT Y R. ABIZANDA CAMPOS
}

Servei de Medicina Intensiva. Hospital Universitario Asociado General de Castelló. Castellón. España.

A mediados de 2005 ha aparecido la publicación de Simplified Acute Physiology Score 3 (SAPS 3) (S, $^{1,}$ última versión del sistema SAPS, desarrollada a partir de un estudio multicéntrico, internacional, coordinado por el Health Services and Research Outcome (HSRO), grupo de trabajo de la European Society of Intensive Care Medicine (ESICM). Las novedades que representa SAPS 3 merecen cierto comentario independiente del hecho de que su uso sea libre de costes, al no estar protegido por copyright, como corresponde a un instrumento científico puesto al servicio de la comunidad médica internacional, y a diferencia de su más próximo «competidor», la versión 3 de Acute Physiology and Chronic Health Evaluation (APACHE 3), que en su diseño internacional está protegida por copyright y sólo es utilizable mediante un pago (no pequeño) que permite el acceso al software. Existe una versión española de APACHE $3^{4}$, libre de uso, cuyo desarrollo está adaptado a características específicas de los pacientes españoles.

Con respecto al desarrollo metodológico de SAPS 3 hay poco que decir. Se trata de un método de regresión logística, en el que los coeficientes de los distintos factores (la ecuación original aparece en la publicación) están ponderados según su importancia en la capacidad predictiva total. Su capacidad de discriminación global entre predichos fallecidos y falsos predichos fallecidos ha sido comprobada mediante el cálculo del área bajo la curva de características operativas del receptor (curva ROC) $)^{5}$, y su calibración (exactitud de predicción a distintas probabilidades de fallecimiento) ha sido comprobada por las pruebas de bondad de ajuste $\mathrm{C}$ y $\mathrm{H}$ de Lemeshow 6 . La relación entre mortalidad real y mortalidad predicha ha sido expresada a través del cálculo de la tasa estan-

Correo electrónico: abizanda_ric@gua.es / rabizandac@terra.es darizada de mortalidad (SMR), y únicamente se echa a faltar la comparación predictiva con otros sistemas al uso, como puede ser SAPS 2, APACHE 2 ó 3, o cualquier otro.

Esta comparación se hubiera establecido mediante el test de concordancia de Bland Altmann ${ }^{7}$, como es habitual, y la posibilidad de interutilizar la predicción de uno de esos sistemas para establecer la probabilidad predicha por otro se hubiera hecho por el cálculo de la ecuación descriptiva de la regresión lineal entre ambos.

Ésta es la metodología habitual, no representa ninguna novedad, y no merece mayor comentario. Como ejemplo de estos planteamientos, Medicina Intensiva pública $^{8}$ un reciente original en el que nuestro grupo aborda la comparación predictiva entre EPEC, SAPS 2 y MPM II 0 sobre la base de los pasos aquí descritos.

Sin embargo hay otros hechos que sí merecen esta atención y muy detallada. Y tienen que ver con las «novedades» que aporta SAPS 3 y en las que radica gran parte de su atractivo.

La primera de estas características es el «timing» de establecimiento del pronóstico, o sea el momento en el que se establece la predicción. SAPS 3 se construye con datos recogidos durante el ingreso inmediato del paciente. Es decir, a la hora de ingreso y tal como es habitual, el sistema no es válido para pacientes individuales, y el riesgo establecido es el grupo de pacientes de las mismas características. Dicho de otra forma, ni este sistema -ni ninguno de los disponibles hasta el momento- permite tomar decisiones individuales, sino que da orientaciones poblacionales, de grupo. No permite pues ni decidir un ingreso particular, ni establecer una estrategia de posible limitación asistencial, pero es, como todos sus «hermanos», una excelente herramienta de control de calidad.

La segunda característica que debe mencionarse es que este sistema recoge (por primera vez) el tiem- 
po de estancia en planta -cualquier planta- antes del ingreso en Unidad de Cuidados Intensivos (UCI). Aborda así el problema del llamado «lead time bias» conocido desde hace años ${ }^{9,10}$ pero no abordado de forma satisfactoria hasta la fecha, con independencia de EPEC, el sistema descrito por Padrón et al, desde Cuba, y validado en un estudio realizado en nuestro país ${ }^{8}$.

Por otro lado, SAPS 3 incorpora a la regresión logística tres tipos de variables distintas relacionadas con el diagnóstico y que condicionan marcadamente el pronóstico (como ya existía la firme sospecha). Estas variables son: a) las comorbilidades existentes, b) el tipo y localización del procedimiento quirúrgico, y c) el tipo de paciente (según el sistema ya utilizado por SAPS 2: quirúrgico urgente o programado y no quirúrgico). Esta novedad sale al paso de la clásica discusión entre sistemas de estimación pronóstica genéricos o específicos, decantándose por la relativa especificidad ligada a la tipología de los pacientes y no a un diagnóstico concreto, como hasta ahora había optado el sistema APACHE.

$\mathrm{Y}$, por fin, dos últimas características a destacar. Una es que la exactitud del sistema está profundamente ligada al sistema sanitario en el que se aplica. Así, existen dos ecuaciones de regresión para SAPS 3. Una genérica, que permite la comparación internacional de los datos, y que sitúa el análisis de prestaciones de una UCI en concreto frente a cualquier UCI representativa de cualquier otro sistema de planificación asistencial. La otra fórmula es específica de área geográfica, habiéndose desarrollado siete formulaciones distintas, donde cada investigador debe ubicarse a la hora de establecer su análisis de calidad, y que recogen la variabilidad introducida en el desarrollo del sistema por el sesgo de los participantes (en número de unidades y número de casos aportados) en el proyecto. Ésta es la consecuencia directa de una investigación en la que la incorporación es individual y de carácter voluntario, como ha sido habitualmente la «norma» hasta ahora y que permite comprobar a través de las distintas SMR cómo el sistema puede «funcionar» de forma muy distinta dependiendo del lugar donde se aplique y de los grupos poblacionales incorporados a su desarrollo, por esa zona geográfica.

Para acabar, debe enfatizarse que SAPS 3 ha estudiado el peso de los distintos componentes de la ecuación (variables relacionadas con la demografía del paciente, variables relacionadas con el diagnóstico y motivo de ingreso en UCI, y variables relacionadas con la alteración fisiopatológica detectada) y que de este estudio se deduce la repercusión que cada uno de estos componentes tiene sobre el peso final comprobándose que los dos primeros componentes son responsables de casi el $75 \%$ de la capacidad de predicción, mientras que la alteración fisiopatológica es responsable tan sólo de un $25 \%$. Este hallazgo puede plantear un cambio conceptual y filosófico importantísimo en los intentos de aplicar la teoría del pronóstico a los pacientes críticos; cuestiona, en gran parte, toda la metodología seguida hasta la fecha en el desarrollo de sistemas de predicción de supervivencia, y justifica el distinto «timing» de aplicación de SAPS 3 con respecto a sus homólogos, como ya se ha mencionado más arriba.

Es evidente que los sistemas de estimación pronóstica tienen una validez limitada en el tiempo. A medida que el desarrollo de la Medicina Intensiva y la capacidad de atención al paciente crítico mejoran, la fiabilidad de los distintos sistemas parece resentirse, y se da la paradoja de que con el paso del tiempo nuestra capacidad asistencial parece ser «mejor» porque los valores de las tasas estandarizadas de mortalidad (resultado real frente a predicción teórica) disminuyen, alejándose del 1. Ésta es una falsa impresión respecto a la fiabilidad de los sistemas de estimación pronóstica. Lo que sucede es que nuestra prestación asistencial mejora en calidad, y al evaluarla mediante sistemas diseñados años atrás, éstos funcionan menos ajustadamente.

Pensamos que, hoy por hoy, SAPS 3 es el mejor sistema de estimación pronóstica de los disponibles al alcance de los especialistas en pacientes críticos. Le llegará su obsolescencia, esperemos que tarde y limitada, y ése será el momento de empezar a pensar en SAPS 4.

\section{BIBLIOGRAFÍA}

1. Metnitz PGH, Moreno RP, Almeida E, Jordan B, Bauer P, Abizanda Campos R, et al. SAPS3: from evaluation of the patients to evaluation of intensive care unit. Part I: Objectives, methods and cohort description. Intensive Care Med. 2005;31:1336-44.

2. Moreno RP, Metnitz PGH, Almeida E, Jordan B, Bauer P, Abizanda Campos R, et al. SAPS 3: from evaluation of patients to evaluation of intensive care unit. Part II: Development of a prognostic model for hospital mortality at ICU admission. Intensive Care Med. 2005;31:1345-55.

3. Knaus WA, Wagner DP, Draper EA, Zimmerman JE, Bergner M, Bastos PG, et al. The APACHE III prognostic system: risk prediction of hospital mortality for critically ill hospitalised adults. Chest. 1991;100:1619-39.

4. Vázquez Mata G, Jiménez Quintana MM, Rivera Fernández R, Bravo M, Aguayo de Hoyos E, Zimmermann J, et al. Evaluación de la gravedad mediante APACHE III en España. Med Clin (Barc). 2001;117:446-51.

5. Hanley JA, McNeil BJ. The Meaning and Use of the area under a receiver operating characteristic (ROC) curve. Radiology. 1981;143:29-36.

6. Lemeshow S, Hosmer DW. A review of Goodness-of-fit Statistics for Use in the development of logistic regression models. Am J Epidemiol. 1982;115:92-106.

7. Bland JM, Altman DG. Measuring agreement in method comparison studies. Stat Methods Med Res. 1999;8:135-60.

8. Abizanda R, Padrón A, Vidal B, Mas S, Berenguer A, Madero J, et al. Estimación pronóstica en enfermos críticos. Validación de un nuevo y sencillo sistema de estimación pronóstica de supervivencia en pacientes ingresados en una Unidad de Cuidados Intensivos. Med Intensiva. 2006;30:101-8.

9. Tunnell RD, Millar BW, Smith GB. The effect of lead time bias on severity of illness scoring, mortality prediction and standarised mortality ratio in intensive care - a pilot study. Anesthesia. 1998;53:1045-53.

10. Dragsted L, Jorgensen J, Jensen NH, Bonsing E, Jacobsen E, Knaus WA, et al. Interhospital comparisons of patient outcome from intensive care: importance of lead-time bias. Crit Care Med. 1989;17:418-22. 